\title{
Clinics in diagnostic imaging (171)
}

Su Kai Gideon Ooi ${ }^{1}$, MBBS, Tien Jin $\underline{T a n}^{2}$, MBBS, FRCR, James Chi Yong $\underline{N g u}^{3}$, MBBS, MRCSEd
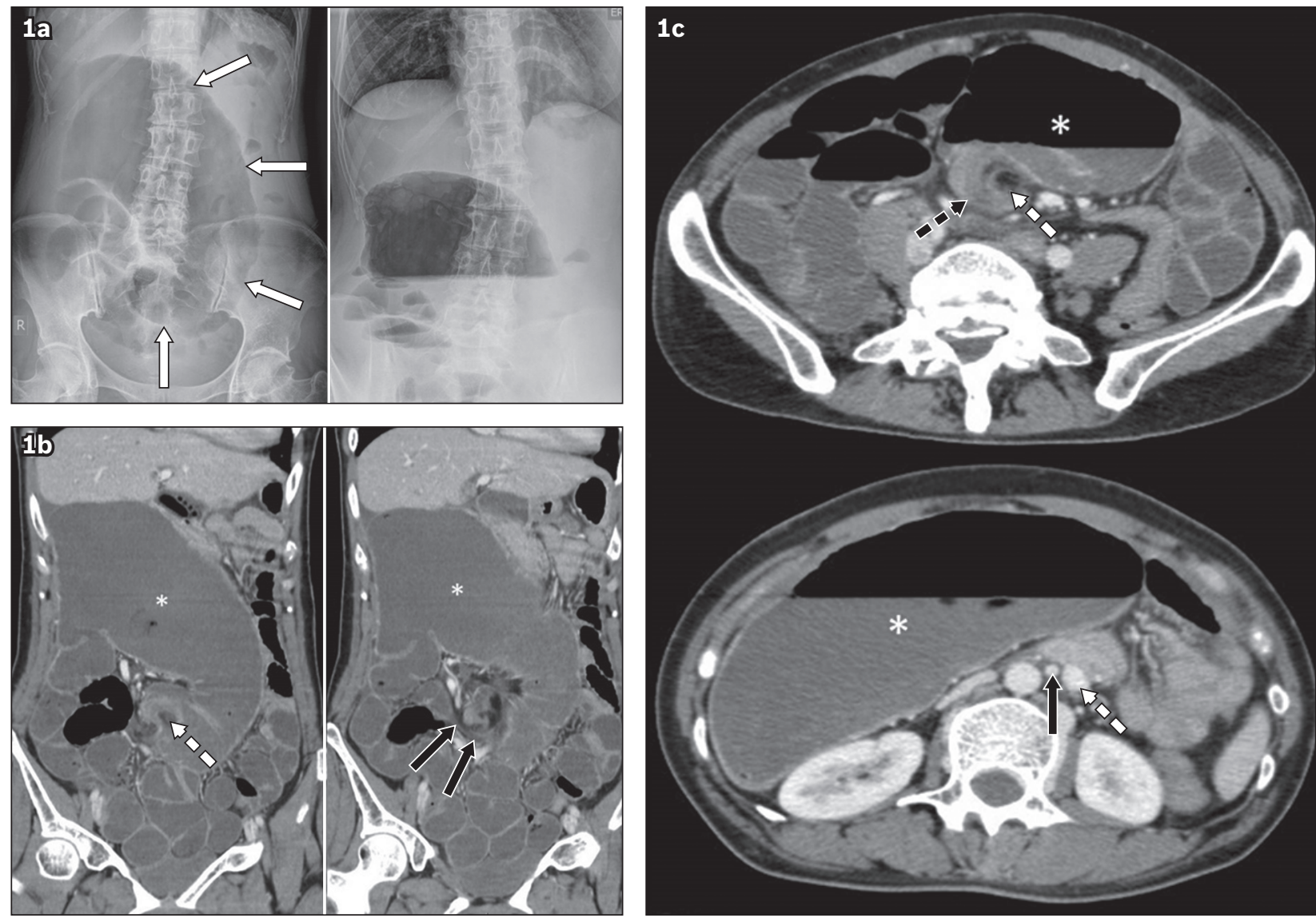

Fig. 1 (a) Supine (left) and erect (right) plain radiographs of the abdomen. Contrast-enhanced (b) coronal and (c) axial CT images of the abdomen and pelvis.

\section{CASE PRESENTATION}

A 46-year-old Chinese woman presented to the emergency department with nausea and abdominal bloating, accompanied by vomiting that had increasingly worsened over the past four days. Apart from previous laparoscopic cholecystectomy and open appendicectomy, she had no other medical comorbidities. Physical examination revealed abdominal distension with central tenderness and active bowel sounds. There was no rebound tenderness or abdominal guarding. She was afebrile and her haemodynamic parameters were stable. Initial blood investigations revealed an elevated total white blood cell count of 15.9 (normal range [NR] 4.0-10.0) $\times 10^{3} / \mathrm{uL}$ and a raised serum lactate level of 2.28 (NR 0.5-1.0) mmol/L. Arterial blood gas results were $\mathrm{pH} 7.45$ (NR 7.35-7.45); partial pressure of carbon dioxide $\left(\mathrm{pCO}_{2}\right) 28.6$ (NR 35.0-45.0) mmHg; and standard bicarbonate 22.2 (NR 21.0-27.0) mmol/L, suggesting the presence of partially compensated primary respiratory alkalosis with no evidence of primary metabolic acidosis.

Plain radiography of the abdomen (Fig. 1a) was performed at the emergency department. What do the radiographs show? Based on the patient's clinical presentation and radiographic findings, computed tomography (CT) of the abdomen and pelvis (Figs. 1b \& c) was also performed. What diagnosis is confirmed on subsequent $\mathrm{CT}$ ? What underlying condition does the patient have?

'Diagnostic Radiology Programme, SingHealth Residency, 2Department of Diagnostic Radiology, 3Department of General Surgery, Changi General Hospital, Singapore Correspondence: Dr Gideon Ooi Su Kai, Resident, Diagnostic Radiology Programme, SingHealth Residency, GME Office, 167 Jalan Bukit Merah \#17-10 Tower 5, Singapore 150167.Gideon.ooi@mohh.com.sg 


\section{IMAGE INTERPRETATION}

Plain radiographs (Fig. 1a) show a grossly distended large bowel with its long axis extending from the right lower abdomen toward the epigastrium (white arrows), and an intraluminal air-fluid level. Distension of a few small bowel loops in the central abdomen can also be seen. These findings are suspicious for an acute caecal volvulus. CT images (Figs. $1 \mathrm{~b} \& \mathrm{c}$ ) confirm the presence of a dilated caecum (indicated by *), while spiralling of the twisted mesenteric vessels and fat at the point of torsion give rise to a 'whirl' sign (white broken arrow, Fig. 1b). Both the afferent (black arrows, Fig. 1b) and efferent limbs (black broken arrow, Fig. 1c) of the caecal volvulus taper and converge at the site of torsion in the central abdomen. The bowel loops are dilated, but do not show evidence of established ischaemia or perforation. The superior mesenteric vein (white broken arrows, Fig. 1c) also lies to the left of the superior mesenteric artery (black arrow, Fig. 1c), contrary to normal anatomy. This is in keeping with intestinal malrotation.

\section{DIAGNOSIS}

Caecal volvulus with underlying intestinal malrotation.

\section{CLINICAL COURSE}

The patient was operated on emergently due to the risk of colonic perforation. The findings of caecal volvulus with serosal tears and punctate perforation of the ascending colon were confirmed intraoperatively (Fig. 2). The midgut was found to be non-rotated; there was a $180^{\circ}$ anti-clockwise rotation of the mesentery toward the caecum, as well as a lack of peritoneal fixation of the right hemicolon and duodenum. The decision was made to perform a right hemicolectomy. The small bowel was decompressed via an enterotomy, and a side-to-side ileocolic stapled anastomosis was performed. The patient recovered uneventfully following surgery and was discharged on postoperative Day 6 .

\section{DISCUSSION}

Caecal volvulus is an uncommon cause of bowel obstruction, with an estimated incidence of 2.8-7.1 per million people per year, accounting for $1 \%-1.5 \%$ of all adult intestinal obstructions and $25 \%-40 \%$ of all cases of colonic volvulus. ${ }^{(1)}$ Although the aetiology of caecal volvulus is often thought to be complex and multifactorial, excessive caecal mobility is a commonly implicated factor. ${ }^{(2,3)}$ This is thought to be due to incomplete intestinal rotation during embryonic development, resulting in inadequate or abnormal fixation of the right hemicolon. ${ }^{(4)}$ Other contributory factors include prior abdominal surgery, late-term pregnancy, and high fibre intake or chronic constipation. These factors presumably cause increased caecal displacement or hyperperistalsis. ${ }^{(2,5)}$

Clinically, patients tend to present with recurrent intermittent abdominal pain or acute intestinal obstruction. Close to half of patients who present with acute obstruction due to caecal volvulus have a history of preceding recurrent intermittent abdominal pain that is localised to the right lower quadrant of the abdomen. They may develop high-pitched bowel sounds

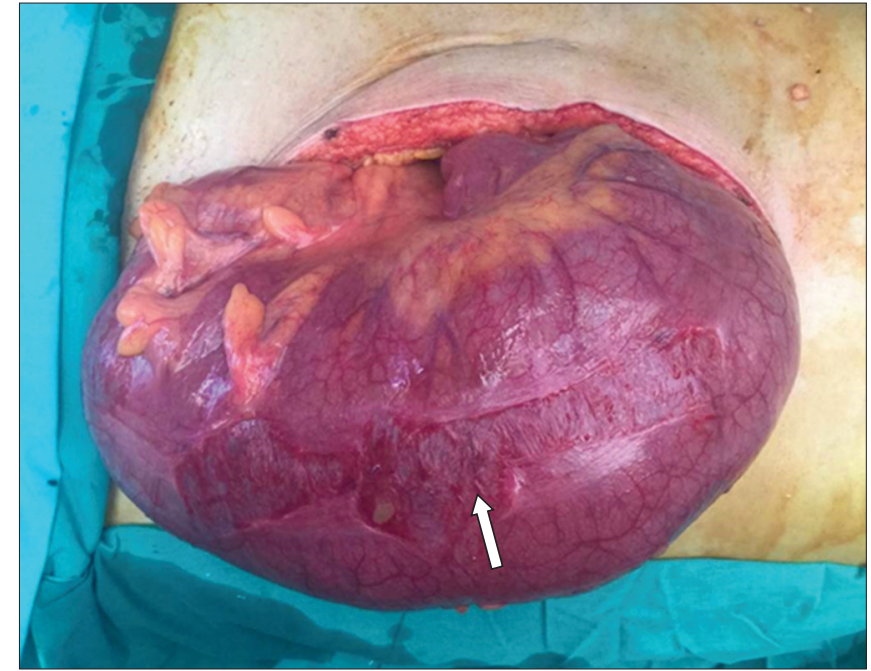

Fig. 2 Intraoperative photograph of the grossly dilated caecum with evidence of a serosal tear (white arrow).

and focal tenderness during symptomatic episodes that resolve spontaneously. Clinically, acute intestinal obstruction due to caecal volvulus is often indistinguishable from other causes of acute bowel obstruction. Hence, imaging investigations are useful adjuncts in diagnosis.

Although CT is often the best imaging tool in the diagnosis of caecal volvulus, ${ }^{(5)}$ plain radiography is usually the first-line imaging investigation used in the evaluation of central abdominal pain. ${ }^{(2)}$ Therefore, it is important to recognise radiographic abnormalities that are identifiable in nearly all patients who present acutely with intestinal obstruction due to caecal volvulus. These include caecal dilatation (seen in $98 \%-100 \%$ of cases), a dominant intraluminal air-fluid level $(72 \%-88 \%)$, dilatation of the small bowel $(42 \%-55 \%)$ and a paucity of gas in the distal colon $(82 \%) .{ }^{(1)}$ Despite the high sensitivity of radiographic evaluation, it lacks the specificity conferred by CT imaging in the diagnosis of caecal volvulus, and patients are not uncommonly misdiagnosed as having small bowel obstruction.

The 'CT coffee bean', 'bird beak' and 'whirl' signs are three common CT findings that are associated with acute caecal volvulus. The 'CT coffee bean' sign generally refers to an axial view of a dilated caecum filled with air and fluid that may be visualised anywhere within the abdominal cavity. It should not be confused with the radiographic 'coffee bean' sign associated with sigmoid volvulus. The 'bird beak' sign refers to progressively tapering efferent and afferent bowel loops pointing to the site of torsion, which resemble bird beaks. The 'whirl' sign on a CT image describes a soft-tissue mass with internal architecture that contains swirling strands of soft tissue and fat attenuation. In the setting of acute caecal volvulus, the 'whirl' is composed of a spiralled loop of collapsed caecum with low-attenuating fatty mesentery and engorged mesenteric vessels. ${ }^{(6)} \mathrm{A}$ variant of caecal volvulus called the caecal bascule has also been described. This is a rare form of caecal obstruction caused by folding of the caecum anterior to the ascending colon, resulting in a 'flap valve' that occludes caecal emptying without true twisting; caecal bascule is 

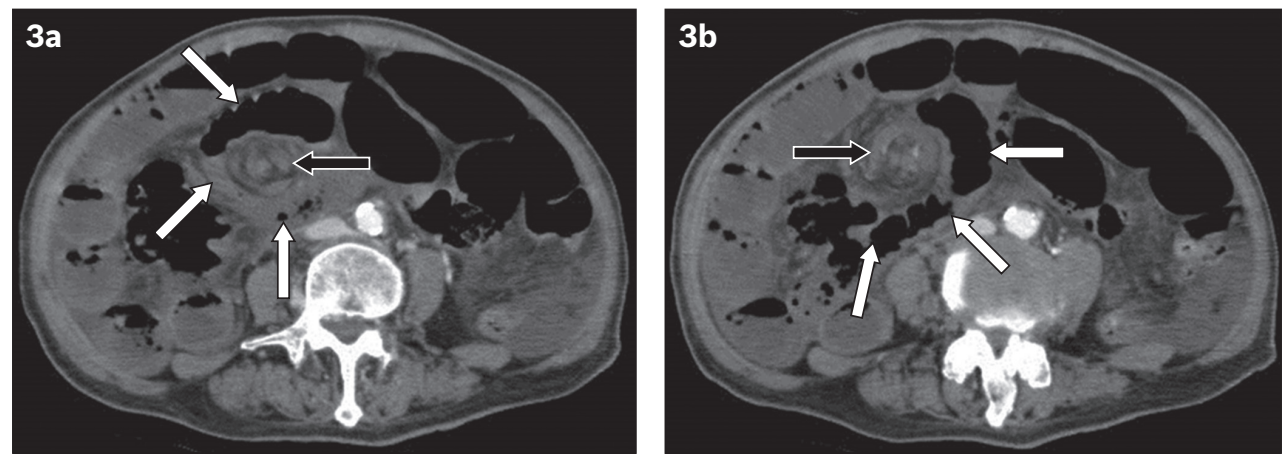

Fig. 3 Intestinal malrotation complicated by midgut volvulus and bowel ischaemia in a 46-year-old woman who presented with abdominal distension and vomiting of four days' duration. (a \& b) Contrast-enhanced axial CT images show swirling of the mesenteric root vessels (black arrows) and small bowel (white arrows) at the site of the volvulus. Note the non-enhancing mesenteric root vessels at the site of the volvulus, as well as diffuse non-enhancement of the small bowel loops, which is consistent with extensive bowel ischaemia.

responsible for $10 \%$ of all cases of caecal obstruction. ${ }^{(7,8)}$ Patients with caecal bascule tend to have a better prognosis and lower incidence of vascular compromise of the bowel. An abnormal positional relationship between the superior mesenteric vein and artery, indicative of intestinal malrotation, is also identifiable on $\mathrm{CT} .{ }^{(9)}$ Contrary to normal anatomy, the vein is located to the left of the artery.

Treatment options for caecal volvulus include endoscopic reduction and surgery. Endoscopic reduction only has a success rate of approximately $30 \%$ and is generally considered ineffective. ${ }^{(10)}$ The general consensus, therefore, is that surgical management of caecal volvulus is recommended, with the exact surgical approach depending on the intraoperative findings. Bowel resection is preferred when there is evidence of bowel gangrene or impending perforation, as seen in our patient. Non-resectional surgery, such as caecopexy or caecostomy, is generally less invasive and may be considered in the absence of bowel ischaemia. Residual hypermobility of the right hemicolon after caecal-sparing surgery has been implicated as a cause for the high recurrence rates of caecal volvulus, which were previously reported to be up to $40 \%$ for caecopexy and $33 \%$ for caecostomy tube placement. ${ }^{(1)}$

Intestinal malrotation is also a major predisposing factor for midgut volvulus. As compared to cases of caecal volvulus in which there is inadequate or abnormal fixation of the right hemicolon, midgut volvulus occurs when there is abnormal fixation of the small bowel mesentery, resulting in an abnormally short mesenteric root. ${ }^{(11)}$ Plain radiography findings in cases of midgut volvulus are nonspecific and seldom helpful in establishing a definitive diagnosis. Fluoroscopic upper gastrointestinal and small bowel studies are useful in demonstrating the characteristic features of midgut volvulus, namely the abnormal position of the small bowel in the right side of the abdomen and that of the ligament of Treitz (indicative of malrotation), as well as the corkscrew-like appearance of the small bowel if midgut volvulus is present. ${ }^{(12)}$ Knowledge of the CT findings of midgut volvulus is also important; the preoperative diagnosis is often made based on cross-sectional imaging, given that most patients present with nonspecific symptoms. Aside from the aforementioned abnormal positions of the small bowel and ligament of Treitz, other characteristic CT findings include swirling of the mesenteric root vessels at the site of the volvulus (Fig. 3) and an abnormal relationship between the superior mesenteric artery and vein. ${ }^{(8,13)}$ Twisting of the small bowel about its mesentery also predisposes an individual to ischaemia of the bowel (Fig. 3). Midgut volvulus often occurs early in life and surgery is usually performed to repair the malrotation.

In conclusion, it is imperative to recognise the imaging features of caecal volvulus early, so as to ensure appropriate and expedient management, and thus reduce patient morbidity from potential complications. CT is more specific when compared to radiography and has become the imaging modality of choice for establishing a preoperative diagnosis of caecal volvulus and distinguishing this condition from other causes of intestinal obstruction.

ABSTRACT A 46-year-old Chinese woman with a history of cholecystectomy and appendicectomy presented to the emergency department with symptoms of intestinal obstruction. Physical examination revealed central abdominal tenderness but no clinical features of peritonism. Plain radiography of the abdomen revealed a grossly distended large bowel loop with the long axis extending from the right lower abdomen toward the epigastrium, and an intraluminal air-fluid level. These findings were suspicious for an acute caecal volvulus, which was confirmed on subsequent contrast-enhanced computed tomography (CT) of the abdomen and pelvis. CT demonstrated an abnormal positional relationship between the superior mesenteric vein and artery, indicative of an underlying intestinal malrotation. This case highlights the utility of preoperative imaging in establishing the diagnosis of an uncommon cause of bowel obstruction. It also shows the importance of recognising the characteristic imaging features early, so as to ensure appropriate and expedient management, thus reducing patient morbidity arising from complications.

Keywords: abdominal radiography, computed tomography, intestinal obstruction, malrotation, volvulus 


\section{REFERENCES}

1. Consorti ET, Liu TH. Diagnosis and treatment of caecal volvulus. Postgrad Med J 2005; 81:772-6.

2. Rabinovici R, Simansky DA, Kaplan O, Mavor E, Manny J. Cecal volvulus. Dis Colon Rectum 1990; 33:765-9.

3. Wolfer JA, Beaton LE, Anson BJ. Volvulus of the cecum. Anatomical factors in its etiology: report of case. Surg Gynecol Obstet 1942; 74:882-94.

4. Donhauser JL, Atwell S. Volvulus of the caecum with a review of 100 cases in the literature and a report of six new cases. Arch Surg 1949; 58:129-48.

5. Rosenblat JM, Rozenblit AM, Wolf EL, et al. Findings of cecal volvulus at CT. Radiology 2010; 256:169-75.

6. Moore CJ, Corl FM, Fishman EK. CT of cecal volvulus: unraveling the image. AJR Am J Roentgenol 2001; 177:95-8.
7. Madiba TE, Thomson SR. The management of cecal volvulus. Dis Colon Rectum 2002; 45:264-7.

8. Bobroff LM, Messinger NH, Subbarao K, Beneventano TC. The cecal bascule. Am J Roentgenol Radium Ther Nucl Med 1972; 115:249-52.

9. Bernstein SM, Russ PD. Midgut volvulus: a rare cause of acute abdomen in an adult patient. AJR Am J Roentgenol 1998; 171:639-41.

10. Theuer C, Cheadle WG. Volvulus of the colon. Am Surg 1991; 57:145-50.

11. Peterson CM, Anderson JS, Hara AK, Carenza JW, Menias CO. Volvulus of the gastrointestinal tract: appearances at multimodality imaging. Radiographics 2009; 29:1281-93.

12. McAlister WH, Kronemer KA. Emergency gastrointestinal radiology of the newborn. Radiol Clin North Am 1996; 34:819-44.

13. Fisher JK. Computed tomographic diagnosis of volvulus in intestinal malrotation. Radiology 1981; 140:145-6. 


\section{SINGAPORE MEDICAL COUNCIL CATEGORY 3B CME PROGRAMME} (Code SMJ 201611B)

Question 1. Regarding caecal volvulus:

(a) It accounts for $1 \%-1.5 \%$ of all adult intestinal obstructions.

(b) It accounts for $75 \%-90 \%$ of all cases of colonic volvulus.

(c) It may present with either recurrent intermittent abdominal pain or acute intestinal obstruction.

(d) It is often clinically indistinguishable from other causes of acute bowel obstruction.

Question 2. Predisposing factors for developing caecal volvulus include:
(a) Excessive caecal mobility.
(b) Intestinal malrotation.
(c) Early pregnancy.
(d) Chronic constipation.

Question 3. Regarding the imaging evaluation of caecal volvulus:

(a) Plain radiography is the best imaging tool in the diagnosis of caecal volvulus.

(b) Computed tomography (CT) is often the first-line imaging investigation.

(c) Plain radiography is both highly sensitive and specific in the diagnosis of caecal volvulus.

(d) With radiographic evaluation, patients are not uncommonly misdiagnosed as having small bowel obstruction.

Question 4. Imaging features of caecal volvulus on plain radiography include:
(a) Caecal dilatation.
(b) Dominant intraluminal air-fluid level related to the dilated caecum.
(c) Dilatation of small bowel loops.
(d) Gaseous distension of the distal colon.

Question 5. Regarding the CT imaging features of caecal volvulus:

(a) The 'CT coffee bean' sign refers to a dilated sigmoid colon filled with air and fluid.

(b) The 'bird beak' sign on CT refers to progressively tapering efferent and afferent bowel loops that point to the site of torsion.

(c) The 'whirl' sign is composed of a spiralled loop of collapsed caecum, with low-attenuation fatty mesentery and engorged mesenteric vessels.

(d) The superior mesenteric vein lies to the left of the superior mesenteric artery in cases of intestinal malrotation.

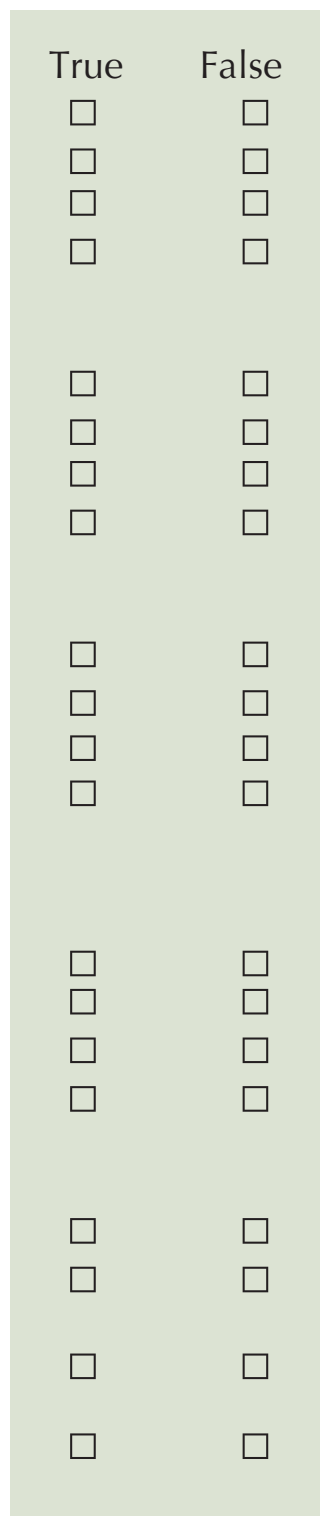

\section{Doctor's particulars:}

Name in full

MCR number

Email address

\section{SUBMISSION INSTRUCTIONS:}

(1) Visit the SMJ website: http://www.smj.org.sg/current-issue and select the appropriate set of questions. (2) Provide your name, email address and MCR number. (3) Select your answers and click "Submit".

RESULTS:

(1) Answers will be published online in the SMJ January 2017 issue. (2) The MCR numbers of successful candidates will be posted online at the SMJ website by 30 December 2016. (3) Passing mark is $60 \%$. No mark will be deducted for incorrect answers. (4) The SMJ editorial office will submit the list of successful candidates to the Singapore Medical Council. (5) One CME point is awarded for successful candidates.

Deadline for submission: (November 2016 SMJ 3B CME programme): 12 noon, 23 December 2016. 